Check for updates

Cite this: RSC Adv., 2018, 8, 36791

Received 25th May 2018

Accepted 14th October 2018

DOI: $10.1039 / \mathrm{c} 8 \mathrm{ra0} 4470 \mathrm{~h}$

rsc.li/rsc-advances

\section{Development of banana (Musa balbisiana) pseudo stem fiber as a surgical bio-tool to avert post- operative wound infections $\dagger$}

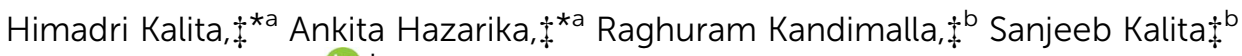 \\ and Rajlakshmi Devi (iD *a
}

\begin{abstract}
The search to develop an ideal suture material encourages us to explore novel suture biomaterials with superior characteristics to the current commercially available products. Surgical sutures play a crucial role in the development of post-operative wound infection by acting as a substrate for biofilm formation which leads to dehisced wounds. In this context, the present invention meets this need by fabricating banana (Musa balbisiana) fibre into an advanced antimicrobials releasing suture biomaterial (BSc) for the prevention of post-operative wound infection. Suture material developed from banana pseudo stem fiber was impregnated with chloramphenicol, clotrimazole and growth factors with the aid of a hydrogel system. The fabricated suture material was found to be biocompatible towards human erythrocytes and L929 mouse fibroblast cells. BSc exhibited promising physico-chemical characteristics which were comparable to the commercially available Bombyx mori silk fibroin (BMSF) suture. BSc displayed a biphasic release pattern with sustained release of chloramphenicol for up to $140 \mathrm{~h}$. Apart from being environment friendly and having a facile fabrication method, this advanced suture biomaterial showed broad spectrum in vitro antimicrobial activity against bacterial and fungal pathogens. BSc successfully impeded biofilm formation on its surface, as is evident from the confocal microscopy analysis. This contributes to superior wound healing efficacy in terms of reduced microbial burden and a subsequent decrease in the inflammatory cytokine levels. Histopathological observations further supported the pronounced healing efficacy of BSc sutured wounds. The findings of this study establish the banana pseudo stem fiber as a novel advanced suture biomaterial to prevent post-operative wound infections.
\end{abstract}

\section{Introduction}

Suture material of natural or synthetic origin can be used for tissue ligation during surgery. Mechanical stability, tissue compatibility, thermal stability, and superior tensile strength are the prerequisites for successful tissue adherence at the healing site. ${ }^{1}$ These properties depend on the chemical composition of the suture and the surface impregnated additives. ${ }^{1,2}$ An ideal suture material possessing all of these essential characteristics has not been realized to date. Knot security, tissue reactivity, and tensile strength are the key disadvantages associated with the commercially available suture materials., Mitigation of the shortcomings of commercially available

${ }^{a}$ Life Sciences Division, Institute of Advanced Study in Science and Technology, Guwahati, Assam, India. E-mail: kalitahimadri123@gmail.com; ankitahazarikabiotech@gmail.com; Tel: +91-9706107073; +91-9706053605

${ }^{b}$ Drug Discovery Laboratory, Institute of Advanced Study in Science and Technology, Guwahati, Assam, 781035, India. E-mail: biochemistry.iasst@gmail.com; Tel: +919706033567

† Electronic supplementary information (ESI) available. See DOI: 10.1039/c8ra04470h

\$ First authors. sutures encouraged us to develop a novel suture biomaterial with superior characteristics. Plant originated fibers are biocompatible and can be developed as a suture material with lower costs, that are eco-friendly and have a facile fabrication process. ${ }^{5}$ Bananas (Musa balbisiana, family: Musaceae) are mainly available in India, South Asia, Southeast Asia, and Southern China. India is the largest producer of bananas, accounting for 16 million tons per year. Large-scale production of fiber from bananas could tremendously contribute towards the uptake of a banana fiber based industrial venture. ${ }^{6}$ After harvesting the fruits, the discarded waste of the pseudo stem is processed for banana fiber preparation, which serves as a prospective raw material for textiles, handicrafts or the banana paper manufacturing industry. ${ }^{6}$ Banana fibers are light weight, have soft fibers, ${ }^{6}$ and are mainly composed of cellulose, lignin, hemicelluloses, and pectin, and have microfibrillar angles which give superior tensile properties. ${ }^{7}$

An ideal suture material should have an antimicrobial property, which can speed up the healing process, and maintain a sustained drug release to the wound area. Post-operative wound infections remains to be a predominant factor contributing to delayed wound healing processes which leads to 
prolonged hospital stays. ${ }^{\mathbf{8} 9}$ Surgical sutures are critical in the settlement of microbial colonies and subsequent establishing of infections. Microbial pathogens exploit the suture surface as a substratum for biofilm formation and spread towards deeper tissues through capillary action. ${ }^{1}$ To prevent suture mediated surgical site infections (SSI), antimicrobial coated sutures with sustained release properties are a valuable alternative strategy. Antibiotics can be applied to sutures in two different ways: passive coating, based on cationic biopolymers that prevent the attachment of bacteria to the suture biomaterial, and active coatings that release drugs into the tissue to inhibit bacterial growth. ${ }^{\mathbf{1 0 , 1 1}}$ Although active strategies are more effective; passive strategies are believed to be more biocompatible than the former. Efficient antimicrobial activity with a profound biofilm obstruction ability can be achieved by coating the biomaterial with a broad spectrum antimicrobial agent like chloramphenicol and clotrimazole, which are active against different wound specific microbes such as Staphylococcus aureus, Staphylococcus epidermidis, and Candida albicans. ${ }^{12}$ Along with antimicrobial agents, impregnation of growth factors in biomedical devices has also been reported to prevent the formation of biofilms. ${ }^{1}$ However, there has been less significant progress in the development of suture material with sustained drug release for longer duration and efficient wound healing. ${ }^{13}$ The healing process can be further accelerated by using growth factors and tissue regenerative phyto-constituents on the suture surface. ${ }^{\mathbf{1 1 4}}$ However, there is not a single suture material that is commercially available with advanced antimicrobial, inherent healing, and controlled drug release properties. The natural hydrogel system, which possesses inherent wound healing characteristics was used in this study to impregnate the suture material with antimicrobial agents.

The present study aims to develop a biocompatible, cost effective suture biomaterial from the waste material of banana pseudo stems by imparting antimicrobial functionalities onto it to achieve accelerated healing of post-operative wounds. The broad spectrum antimicrobial property was achieved by impregnating the suture with antimicrobial drugs (chloramphenicol and clotrimazole) and growth factors (nerve and epithelial growth factors) with the aid of an aloe-vera (AV) and gum acacia (GA) based hydrogel system.

\section{Materials and method}

\subsection{Materials}

Banana (Musa balbisiana) fiber was obtained from Ramie research station $\left(22^{\circ} 3^{\prime} \mathrm{N}, 84^{\circ} 24^{\prime} \mathrm{E}\right)$, Sorbhog, Assam, India. All of the therapeutic molecules and chemicals used in this study were procured from Sigma Aldrich, USA and Merck, Germany. ELISA kits (IL-1 $\beta$ \& TNF- $\alpha$ ) were procured from R\&D systems, USA. The LIVE/DEAD BacLight bacterial viability kit was purchased from Life technologies, USA.

\subsection{Methods}

2.2.1. Suture fabrication and drug impregnation. Raw banana fiber was washed thoroughly to remove the dirt, air dried, and then degummed in $2 \% \mathrm{NaOH}$ solution (at a fiber to $\mathrm{NaOH}$ ratio of $1: 7$ ) at $95{ }^{\circ} \mathrm{C}$ for $120 \mathrm{~min}$. After degumming the fiber was washed thoroughly in distilled water to remove the traces of $\mathrm{NaOH}$ and dried at $37{ }^{\circ} \mathrm{C}$ at $65 \%$ relative humidity. Furthermore, the degummed fiber was braided using a manual braiding technique, which was used for the preparation of banana suture (BS). The braided suture was further sterilized using the moist heat sterilization method in an auto clave $\left(121{ }^{\circ} \mathrm{C}, 100 \mathrm{kPa}\right.$ pressure) for $20 \mathrm{~min}$ and kept in a desiccator prior to use.

The coating material was prepared by dissolving $4.4 \%$ of the antimicrobial agents (chloramphenicol and clotrimazole at $9: 1$ ), nerve growth factor $\left(250 \mathrm{ng} \mathrm{ml}^{-1}\right.$ ) and epithelial growth factor $\left(1 \mathrm{ng} \mathrm{ml}{ }^{-1}\right.$ ) (Sigma, USA) in a mixture of $0.1 \%$ hydrogel base. The hydrogel consisted of AV and GA. AV is a semi liquid gel, whereas GA is a natural adhesive agent, together both the materials help the successful impregnation of drugs onto the surface of the suture material. The braided, sterilized banana suture was dip-coated in the coating material and dried at $37{ }^{\circ} \mathrm{C}$ for $24 \mathrm{~h}$ to form the advanced antimicrobials releasing suture biomaterial (BSc). The percentage of drug impregnation is calculated from eqn (1).

$$
\% \text { of drug impregnation }=\frac{W_{1}-W_{2}}{W_{1}} \times 100
$$

In which, $W_{1}$ is the total weight of drug (chloramphenicol and clotrimazole, $9: 1$ ) dissolved in the hydrogel; $W_{2}$ is the total weight of the remaining drugs in the hydrogel after coating on the suture (BS). $W_{2}$ is calculated from the concentration values obtained from the calibration curve created using UV spectrophotometric analysis of the samples.

2.2.2. Physico-chemical characterization of the BS and BSc. The surface morphology of the raw, degummed and antimicrobial impregnated banana fibers was observed using a field emission scanning electron microscope (FESEM) (Carl Zeiss, SIGMAVP, Japan). The elemental analyses of the above mentioned fibers were determined by using Energy-dispersive X-ray spectroscopy (EDX) (INCA X-Max 250). Functional group analysis of all the fibers (raw, BS and BSc) was performed using attenuated total reflection-Fourier transform infrared spectra (ATR-FTIR) (NICOLET, Thermo Scientific, USA) and compared with the commercially available suture material Bombyx mori silk fibroin (BMSF). Thermo gravimetric analysis (TGA) (Perkin Elmer TGA-4000, USA) was used to determine the thermal stability of the sutures. The tensile strength of the sutures (BMSF suture, raw suture, BS, and BSc) was determined using a tensiometer (Instron tensile tester 3343).

2.2.3. Biocompatibility study. To determine the toxicity of the prepared sutures towards mammalian cells the following tests were conducted.

2.2.3.1. Hemocompatibility. A hemolysis experiment was performed according to a standard protocol. ${ }^{15,16}$ Blood was collected from a healthy human volunteer in sodium citrate siliconized tubes. Informed consent was obtained from the human volunteer before conducting the experiment and the experiment was performed $3 \mathrm{~h}$ after collection of the blood. BMSF, BS, and BSc sutures were separately incubated with $10 \mathrm{ml}$ 
of blood (blood and PBS in 1:9) for $1 \mathrm{~h}$ at $37^{\circ} \mathrm{C}$. After $1 \mathrm{~h}$ the tubes were centrifuged and the optical density of the supernatant was measured at $545 \mathrm{~nm}$ using a UV-spectrophotometer (Shimadzu, UV 1800, Japan). The experiment was performed in triplicate and the percentage of hemolysis was calculated as follows:

$$
\text { Hemolysis }(\%)=\frac{\left(\mathrm{Abs}_{\text {sample }}-\mathrm{Abs}_{-\mathrm{ve} \mathrm{control}}\right)}{\left(\mathrm{Abs}_{+\mathrm{ve} \text { control }}-\mathrm{Abs}_{-\mathrm{ve} \text { control }}\right)} \times 100
$$

2.2.3.2. Effect on morphology of human erythrocyte. Suture materials were incubated with mammalian erythrocyte and its morphology was observed under FESEM. ${ }^{17}$ after the incubation period (from the above experiment) the pellet of erythrocytes was collected and fixed in 3\% glutaraldehyde for $4 \mathrm{~h}$. The fixed cells were washed with $0.2 \mathrm{mM}$ phosphate buffer saline (PBS) and incubated in PBS for $6 \mathrm{~h}$. The incubated cells were subjected to an acetone dehydration process $(30,50,70,90,95$ and $100 \%$ ) and finally treated with tetra methyl saline for 15 min to achieve complete dryness, the powdered erythrocytes were then observed under FESEM.

2.2.3.3. Cytocompatibility. For the cytotoxicity measurement a MTT [3-(4,5-dimethylthiazol-2-yl)-2,5-diphenyltetrazolium bromide] assay was performed against a L929 mouse fibroblast cell line (NCCS, Pune, India). ${ }^{15}$ L929 cells were seeded on 96 well plates $\left(1 \times 10^{4}\right.$ cell per well $)$ in Dulbecco's Modified Eagle's Medium (DMEM) and allowed to attach for $24 \mathrm{~h}$. The cultured cells were then incubated with BMSF, BS, and BSc in DMEM for 24,48 , and $72 \mathrm{~h}$ at $37^{\circ} \mathrm{C}$ in a humidified incubator. This was followed by removal of the medium and adding MTT dye before being incubated again for $4 \mathrm{~h}$. Finally, DMSO was added to each well and the absorbance was measured at $570 \mathrm{~nm}$ using a micro-plate reader. Cells without suture were considered as the positive control. Cell viability was expressed as the percentage of the control according to the following equation:

$$
\text { Viability }(\%)=\frac{N_{\mathrm{t}}}{N_{\mathrm{c}}} \times 100
$$

In which, $N_{\mathrm{t}}$ is the absorbance of the cells treated with sample and $N_{\mathrm{c}}$ is the absorbance of the un-treated cells.

2.2.4. In vitro anti-thrombogenic assay. To assess the in vitro anti-thrombogenic properties, blood was collected from human volunteers. Anti-coagulant (citrate dextrose) treated blood was incubated with BMSF, BS, and BSc in different micro centrifuge tubes for $1 \mathrm{~h}$ at $37^{\circ} \mathrm{C}$. After incubation, $0.2 \mathrm{ml}$ of the blood from each treatment group was poured onto sterilized glass slides and then $0.1 \mathrm{M}$ of calcium chloride was added and it was allowed to clot for 30, 45, and $60 \mathrm{~min}$. After each time interval the clot was fixed with $10 \%$ formaldehyde for $5 \mathrm{~min}$ and washed with distilled water. After washing, the clots were blotted between tissue paper and the weight was measured. Citrate dextrose treated blood without any added samples were also processed using the same protocol and used as a control. ${ }^{18}$

2.2.5. Antimicrobial testing. The antimicrobial activities of the BMSF, BS, and BSc were evaluated against Gram-positive
Staphylococcus aureus (MTCC3160), Gram-negative Escherichia coli (MTCC40), and opportunistic fungus Candida albicans (MTCC3958) by using the standard agar diffusion method and direct contact test method.

In the agar diffusion method, bacterial and fungal cultures were grown on nutrient agar and sabouraud chloramphenicol agar plates respectively. ${ }^{15}$ BMSF, BS, and BSc sutures were placed on the agar plates and incubated at $37{ }^{\circ} \mathrm{C}$ for $24 \mathrm{~h}$ (bacteria) and $28{ }^{\circ} \mathrm{C}$ for $72 \mathrm{~h}$ (fungus). After completion of the incubation period, the zone of inhibition was measured for each suture sample. The experiment was repeated three times and the results are represented as the mean $\pm \mathrm{SD}$.

The direct contact test method was also performed to evaluate the antibacterial ability of BMSF, BS, and BSc. ${ }^{19,20}$ The test materials $(2 \mathrm{~cm})$ were co-inoculated in a nutrient broth medium containing $S$. aureus and $E$. coli at a concentration of $10^{7} \mathrm{CFU}$ $\mathrm{ml}^{-1}$ and incubated at $37{ }^{\circ} \mathrm{C}$ for 21 days. After the incubation period, the bacterial solution $(30 \mu \mathrm{l})$ was collected and inoculated on a nutrient agar plate and incubated at $37{ }^{\circ} \mathrm{C}$ for $24 \mathrm{~h}$. Furthermore, the culture plates were photographed to evaluate the difference in the microbial colony forming unit (CFU).

In order to examine the live and dead bacterial cell population, after the stipulated period of incubation (7 days) the culture medium was removed and suture samples were washed with PBS and a LIVE/DEAD ${ }^{\circledR}$ assay using Backlight ${ }^{\mathrm{TM}}$ (Invitrogen) stain was then performed. During the staining process sutures were kept in the dark for $30 \mathrm{~min}$ and then subjected to confocal microscopy analysis. ${ }^{21}$

2.2.6. In vitro drug release assay. The release profile of the antimicrobial drug (chloramphenicol) was assessed in PBS with pH 6.3, 6.8, and 7.7, corresponding to different skin $\mathrm{pH}$ values from relatively low to high $\mathrm{pH}$ during wound healing. Suture (10 $\mathrm{cm}$ ) was placed in micro centrifuge tubes containing $1.33 \mathrm{ml}$ of the buffers ${ }^{22}$ and kept at $37{ }^{\circ} \mathrm{C}$ under continuous shaking (60 $\mathrm{rpm})$. At different time intervals (24, 48, 72, 96, 120 and $144 \mathrm{~h}$ ), an aliquot of $150 \mu \mathrm{l}$ was recovered to measure the absorbance of each sample by using a Multimode Reader, VARIOSKAN FLASH at different wavelengths of $290 \mathrm{~nm}$ (pH 6.3), $289 \mathrm{~nm}$ (pH 6.8), and $287 \mathrm{~nm}$ ( $\mathrm{pH} 7.7$ ). At the same time an equal volume of PBS was added to the each of the micro centrifuge tubes. The release percentage was calculated as the ratio of drug released at subsequent times to the amount of drug loaded onto the sutures.

\subsubsection{Wound healing efficacy of sutures}

2.2.7.1. Experimental animal. The Institutional Animal Ethics Committee (IAEC) of IASST (Institute of Advanced Study in Science and Technology) approved all of the experimental protocols (IASST/IAEC/2015-16/751). All of the experiments were conducted in accordance with CPCSEA (Committee for the Purpose of Control and Supervision of Experiments on Animals) guidelines. Male Wistar albino rats (10 weeks old, 150-170 g, $n=18$ ) were obtained from the animal house facility of IASST and randomly divided into three experimental groups with six animals each. Animals were housed in individual cages at an ambient temperature of $27 \pm 3{ }^{\circ} \mathrm{C}$ and a relative humidity of $50 \pm 5 \%$ with a 12 h light-dark cycle. Rats were given ad libitum access to food and water. ${ }^{23,24}$ 
2.2.7.2. Animal grouping. BMSF: animals sutured with BMSF and infected with $S$. aureus $\left(2 \times 10^{8}\right.$ cells per $\left.\mathrm{ml}\right) 24 \mathrm{~h}$ after surgery.

BS: animals sutured with BS and infected with $S$. aureus $(2 \times$ $10^{8}$ cells per ml) $24 \mathrm{~h}$ after surgery.

BSc: animals sutured with BSc and infected with $S$. aureus (2 $\times 10^{8}$ cells per ml) $24 \mathrm{~h}$ after surgery.

2.2.7.3. Surgical protocol. Animals were anesthetized with a ketamine (80 $\mathrm{mg} \mathrm{kg}^{-1}$ ) and xylazine $\left(10 \mathrm{mg} \mathrm{kg}^{-1}\right.$ ) cocktail. Hair on the dorsal side of the animals was removed and the skin was cleaned with povidone iodine solution. A $25 \mathrm{~mm}$ thickness wound was incised on the dorsal side using a surgical scalpel. The wounded area was closed with BMSF, BS, or BSc and covered with a cotton gauze. After $24 \mathrm{~h}$ the wounds were infected by inoculation with $2 \times 10^{8}$ cells per $\mathrm{ml}$ of $S$. aureus. On the third, seventh and $14^{\text {th }}$ post-operative day tissue samples were excised, pulverized, and homogenized in sterile PBS and plated on a nutrient agar medium to tally the CFU count of $S$. aureus grown after incubation for $24 \mathrm{~h}$ at $37{ }^{\circ} \mathrm{C} .{ }^{25}$ Blood was collected using the retro orbital route from all of the animals on the third, seventh, and $14^{\text {th }}$ day and serum was separated to measure the inflammatory markers, tumor necrosis factor (TNF- $\alpha$ ) and interleukin (IL)-1 $\beta$ by using ELISA kits from R\&D systems as per the instructions given by the manufacturer (Invitrogen). On the $14^{\text {th }}$ post-surgical day the animals were sacrificed and tissue samples from the surgical site were collected and preserved in 10\% buffered formalin. Fixed tissues were further subjected to the ethanol dehydration process $(30$, 70,90 , and $100 \% \mathrm{v} / \mathrm{v}$ ) and after processing tissue paraffin blocks were prepared. Paraffin embedded tissues were sectioned at 5 $\mu \mathrm{m}$ thickness using a microtome and stained with hematoxylin- eosin and examined under a bright field microscope (LEICA EC3, Germany). ${ }^{26,27}$

2.2.8. Statistical analysis. Statistical analysis was performed using GraphPad Prism version 6 for Windows (San Diego, CA). All of the experimental results were expressed as mean \pm standard error (SE). Statistical significance was tested using Analysis of variance (ANOVA). Multiple comparisons were analyzed using Bonferroni's post hoc test following ANOVA. The threshold for significance was $p<0.05$.

\section{Results and discussion}

\subsection{Physico-chemical characterization of the sutures}

3.1.1. Surface morphological analysis using FESEM. The FESEM images of raw banana fiber, BS and BSc are presented in Fig. 1. Raw banana fiber shows a rough and irregularity surface morphology (Fig. 1A). The roughness is due to presence of lignocellulosic materials like lignin, hemicelluloses, and cellulose,$^{28}$ which are mainly composed of carbon (C) and oxygen (O) and was confirmed using SEM-EDX analysis (Fig. S1a, ESI $\dagger$ ). After alkali treatment, the surface of the BS became smooth, regular and frictionless with a decrease in the diameter of the fiber (Fig. 1B). Alkali treatment resulted in breaking of the intact bundle of the BS into microfibrils and increased the fiber-matrix interfacial adhesion. ${ }^{29}$ After degumming, the surface of BS becomes smooth and frictionless owing to the removal of lignin and hemicellulose. A prominent decrease in the $\mathrm{C}$ and $\mathrm{O}$ in BS compared to the raw fiber was further supported by the results of the SEM-EDX analysis (Fig. S1b, ESI $\dagger$ ). After coating the fiber with the AV-GA hydrogel system the surface of BSc became smooth and uniform throughout its length which was clearly
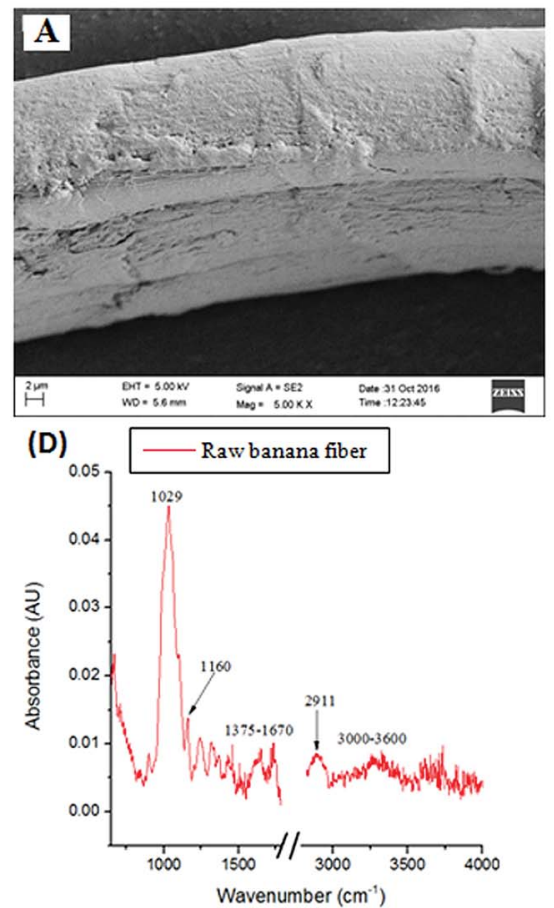
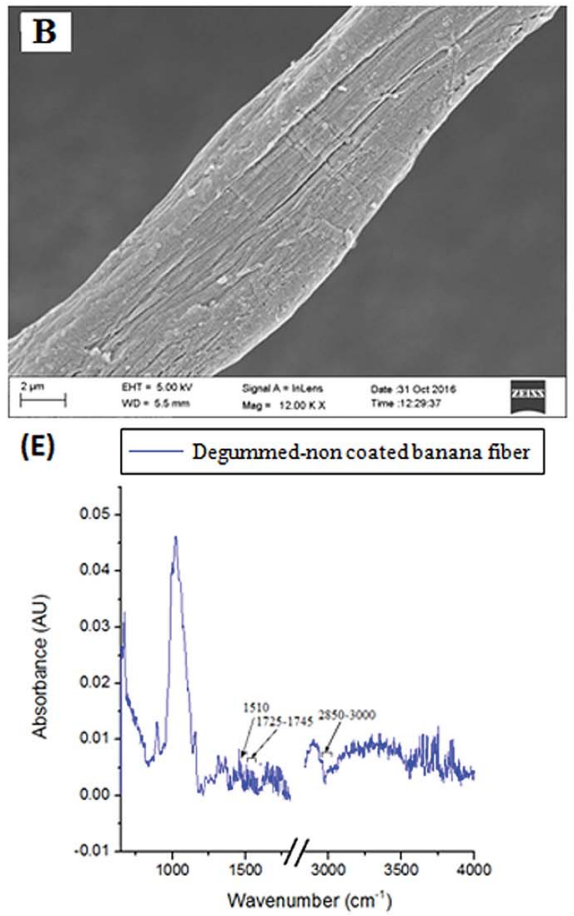
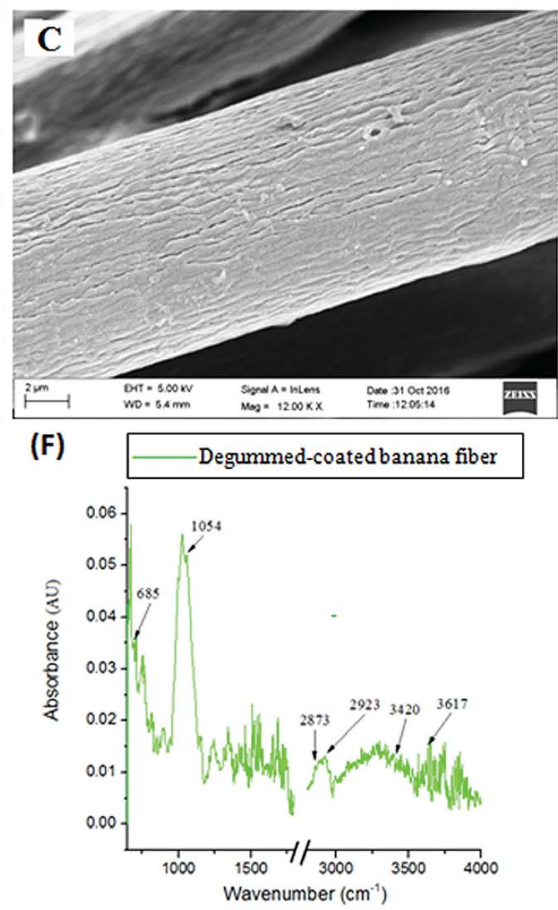

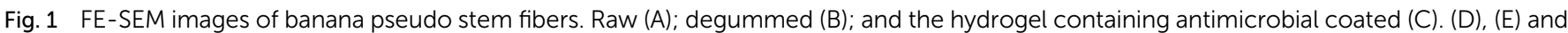
(F) show the ATR-FTIR spectra of the respective fibers at a range of $650-400 \mathrm{~cm}^{-1}$. 
$\pi m a m$ $\mathrm{H}+\mathrm{H} H$ 숭ㅇํ 융 ले observed under FE-SEM (Fig. 1C). This successful impregnation of antimicrobial agents was further supported by the FT-IR data represented in Fig. 1. Detection of magnesium $(\mathrm{Mg})$ and potassium (K) by EDX analysis further supported the successful cladding of the AV-GA hydrogel onto BSc (Fig. S1c, ESI $\dagger$ ). The natural base material $\mathrm{AV}$ contains $\mathrm{Mg}$ and $\mathrm{K}$, which are essential for the proper functioning of different enzymes ${ }^{30}$ responsible for wound remodeling. Chlorine $(\mathrm{Cl})$, the active constituent in chloramphenicol was also detected on the BSc surface. ${ }^{31}$

3.1.2. Functional group characterization. Fourier transform infrared spectroscopy is an appropriate technique to establish the variations introduced by different coatings and chemical treatment of the natural fibers. The FTIR spectra of the raw, BS, and BSc are presented in Fig. 1. The ATR-FTIR analysis of the raw banana fiber showed prominent hemicellulose band intensities as compared to the degummed-noncoated fiber, owing to larger exposure of hemicellulose on the surface. This phenomenon can be attributed to the degumming process of the banana fiber. Both the raw and BS exhibited characteristic vibration bands for vegetables fibers such as sisal, ${ }^{32,33}$ which corresponds to cellulose, lignin, and hemicelluloses. The absorption band at $3600-3100 \mathrm{~cm}^{-1}$ corresponds to the stretching vibrations and other polymeric associations of the $\mathrm{O}-\mathrm{H}$ group in the raw fiber. Similarly, the stretching at $2913 \mathrm{~cm}^{-1}$ is attributed to the $\mathrm{CH}_{2}$ polysaccharide of the raw fiber. The raw fiber also showed absorption peaks at 1654-1327 $\mathrm{cm}^{-1}$ and $1244-1026 \mathrm{~cm}^{-1}$, which correspond to cellulose, hemicelluloses and lignin ${ }^{7}$ respectively. The ATRFTIR of BSc showed major peaks at $688 \mathrm{~cm}^{-1}(\mathrm{C} \equiv \mathrm{C}, \mathrm{C}-\mathrm{H}$ alkynes and alkane respectively), $1054 \mathrm{~cm}^{-1}$ (C-N stretch, aliphatic amine), $2160 \mathrm{~cm}^{-1}$ ( $\mathrm{C} \equiv \mathrm{C}$ stretching), $2111 \mathrm{~cm}^{-1}$ (C $\equiv \mathrm{C}$ stretching), $2860 \mathrm{~cm}^{-1}$ (C-H stretching, alkane), $2923 \mathrm{~cm}^{-1}$ (C-H stretch, alkane), $3429 \mathrm{~cm}^{-1}$ (C-N stretch, amine), and $2921 \mathrm{~cm}^{-1}$ (C-H stretch). Phytochemicals such as aliphatic amines, alkynes, alkanes, carbonyl are mainly present in the innermost part of the plant leaves. ${ }^{34}$ The presence of alkynes, alkanes and amines comes from the natural base material AV-GA and thus confirmed the presence of the coating material on the surface of the BSc.

3.1.3. TGA of the sutures. The TGA of the BSc was carried out to interpret the role of the coating material in the thermal stability of the suture (Fig. S2, ESI $\dagger$ ). The initial weight loss took place at $200{ }^{\circ} \mathrm{C}$ for BS and BSc, which was due to the loss of the water content. The second and final phase of the weight loss occurred between temperatures 300 and $400{ }^{\circ} \mathrm{C}$, which was due to the degradation of hemicelluloses, pectin, and cellulosic components. ${ }^{35}$ The complete degradation occurred at $500{ }^{\circ} \mathrm{C}$ for both BS and BSc. Thus, it can be inferred that the coating material used on the surface of the banana suture did not affect the thermal stability of the suture, as BS and BSc degrade at a similar temperature. In the case of BMSF the initial weight loss owing to the loss of water occurred at $210{ }^{\circ} \mathrm{C}$ and the complete degradation occurred at a temperature of $380^{\circ} \mathrm{C}$. It is evident from the previous study that in the case of Bombyx mori silk the maximum degradation temperature is between 350$400{ }^{\circ} \mathrm{C}$, which is due to the decomposition of the fibroin molecule. ${ }^{36}$ Hence, it is confirmed from the present study that 
both the coated and non-coated banana sutures were more stable at higher temperatures and the results were comparable with those of the BMSF suture.

3.1.4. Tensile property. The tensile properties of the banana fibers improved with alkali treatment and coating. Table 1 shows the tensile properties of the BMSF suture, raw, BS, and BSc sutures. The toughness of the raw, BS and BSc sutures was found to be $0.16 \pm 0.001,0.168 \pm 0.003$, and 0.166 $\pm 0.004 \mathrm{MPa}$ respectively. The commercially available BMSF suture showed a maximum toughness of $0.18 \pm 0.01 \mathrm{MPa}$. From the results it is evident that both the BS and BSc showed almost similar tensile properties to that of the commercially available BMSF suture. No statistically significant difference in tensile properties was observed between the commercially available BMSF suture and the prepared sutures (BS and BSc). It should be noted that a slight variation between our prepared suture and BMSF suture is due to difference in the suture braiding process. Whereas, both BS and BSc were prepared using a manual braiding technique and the latter one was prepared using mechanical braiding. Tensile strength is possibly attributed to the relatively high cellulose content and microfibrillar angle (microfibrillin is the middle layer of a plant secondary cell wall and forms an angle with the cell axis). ${ }^{5}$ The mechanical properties such as the tensile strength and Young's modulus also depends on the fiber-matrix interfacial adhesion, which was improved after the alkali treatment of the fiber. ${ }^{29}$ Mercerization leads to the removal of lignin and hemicelluloses content of the fiber, which in turn leads to a higher cellulose content and improves the tensile strength of the suture. ${ }^{28}$ Furthermore, after impregnation using the natural hydrogel system, the BSc exhibited a superior tensile strength as compared to BS, which is due to the modification of the surface properties of the suture.

\subsection{In vitro biocompatibility evaluation}

3.2.1. Hemocompatibility analysis. Coated and non-coated banana sutures (BS and BSc) were subjected to biocompatibility evaluation. The BS and BSc exhibited very low to mild hemolytic activity (hemolysis percentage) towards the human erythrocyte (Fig. 2F and S3, ESI $\dagger$ ). BSc exhibited a maximum of $0.78 \%$ hemolysis, whereas the permissible limit of hemolysis for blood contacting biomaterials is $5 \% .^{37}$ The components used in the fabrication process of BSc are reported to be hemocompatible and do not induce any adverse effect. The impregnated material contained chloramphenicol and clotrimazole, which are commonly used antibacterial and antifungal agents. Furthermore, the growth factors used were responsible for epithelialization and blood vessel formation, which ultimately increases the healing rate of the wound.$^{38}$ The natural base materials used in this study were bio-safe and are regularly used in therapeutic purposes. ${ }^{14}$

3.2.2. Surface morphology of erythrocyte. The native morphology of erythrocytes shows a biconcave disc structure. On exposure to toxic substances and in abnormal circumstances this may lose its integrity. The FESEM analysis of the erythrocyte after incubation with BS (Fig. 2D), BSc (Fig. 2E) and BMSF (Fig. 2C) did not show any alteration in the morphology, whereas an erythrocyte treated with distilled water showed complete hemolysis (Fig. 2A) and showed a regular morphology after using PBS (Fig. 2B).

3.2.3. Cytocompatibility evaluation. To screen the cytocompatibility of the BSc, BS, and BMSF sutures, an MTT assay
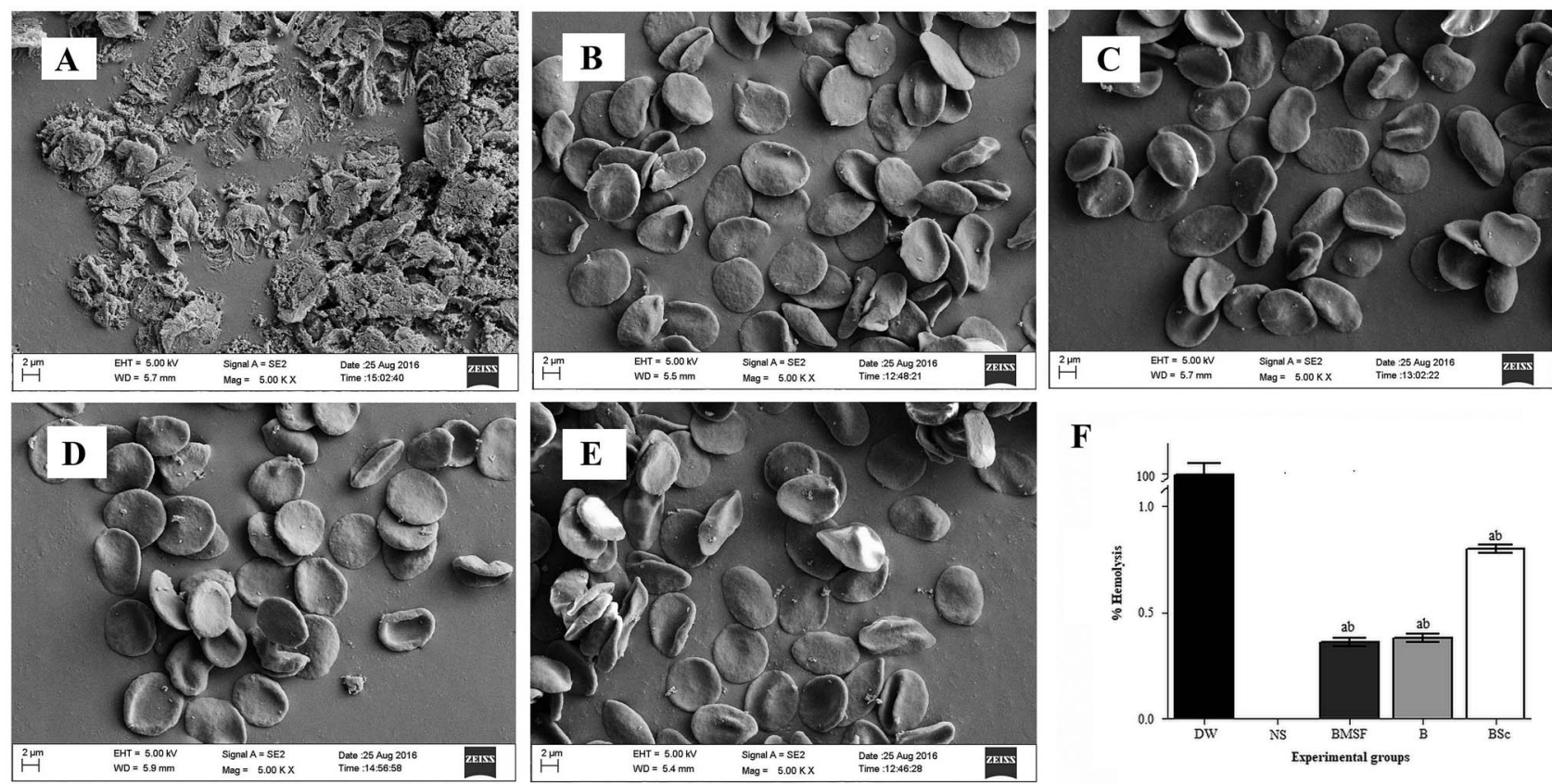

$\mathbf{F}$

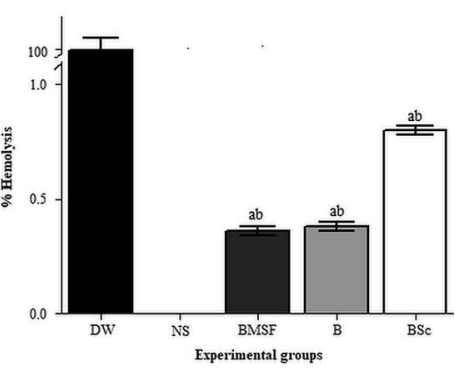

Fig. 2 Morphology of erythrocytes in the presence of different treatments: (A) distilled water (DW); (B) normal saline (NS); (C) BMSF; (D) BS; and (E) BSc under FESEM. (F) Depicts the percentage of hemolysis in the presence of DW, NS, BMSF, BS, and BSc, (a) $p<0.05$ in comparison with DW, and (b) $p<0.05$ in comparison with saline. 
was performed (Fig. S4, ESI $\dagger$ ). After $72 \mathrm{~h}$ of incubation with BMSF and BS the mouse fibroblastic L929 cells exhibited a higher viability percentage of 92.74 and 92.22 respectively. Whereas, BSc showed an $84.33 \%$ cell viability percentage against the mouse L929 cell line. According to ISO norms, a decrease in the cell viability of up to $30 \%$ is considered to be non toxic for medical device materials. Therefore, in this study both BS and BSc are considered to be non toxic towards mammalian cells. Co-existence of the biomaterial along with mammalian cells and tissues is the prime concern in the process of suture development. ${ }^{15}$ The test result shows that both BS and BSc demonstrate cytocompatibility towards mammalian cells.

\subsection{Anti-thrombogenic properties}

An in vitro blood clotting test was performed to evaluate the antithrombogenic properties of the fabricated suture materials. After incubating the sutures with blood, the clotting experiment was conducted and the weight of the clot was measured at different time intervals $(30,45$ and $60 \mathrm{~min}$ ). Fig. S5 (ESI $\dagger$ ) depicts the thrombus formation after incubation of blood with different sutures at different time points. In the control experiment, the thrombus percentage was found to be $2.4 \pm 0.1 \%$ and $80.43 \pm 4 \%$ at 30 and 60 min respectively. Incubation of blood with BS and BSc decreases the percentage of thrombus formation. Whereas the percentage of thrombus formed was found to be $2.1 \pm 0.34 \%$ and $2 \pm 0.6 \%$ at $30 \mathrm{~min}$ and $73 \pm 2 \%$ and $75 \pm 3 \%$ at $60 \mathrm{~min}$ for BS and BSc respectively. It can be concluded from the above findings that BS and BSc were found to be compatible with human erythrocytes.

\subsection{Antimicrobial properties}

The antimicrobial activity of the BMSF, BS, and BSc sutures were evaluated using the agar diffusion test (Fig. 3A). BSc was found to exhibit a significant antimicrobial activity against the tested pathogenic bacteria $E$. coli, $S$. aureus, and opportunistic fungus $C$. albicans with a clear zone of inhibition $(32 \pm 2,29 \pm 3$, and $6 \pm 0.5 \mathrm{~mm}$ respectively), whereas the BS and BMSF sutures did not show any inhibition of bacterial growth on its surface. This clearly indicates the successful impregnation of the antimicrobial drugs on the surface of BSc resulting in the inhibited growth of the tested microorganisms. Chloramphenicol is

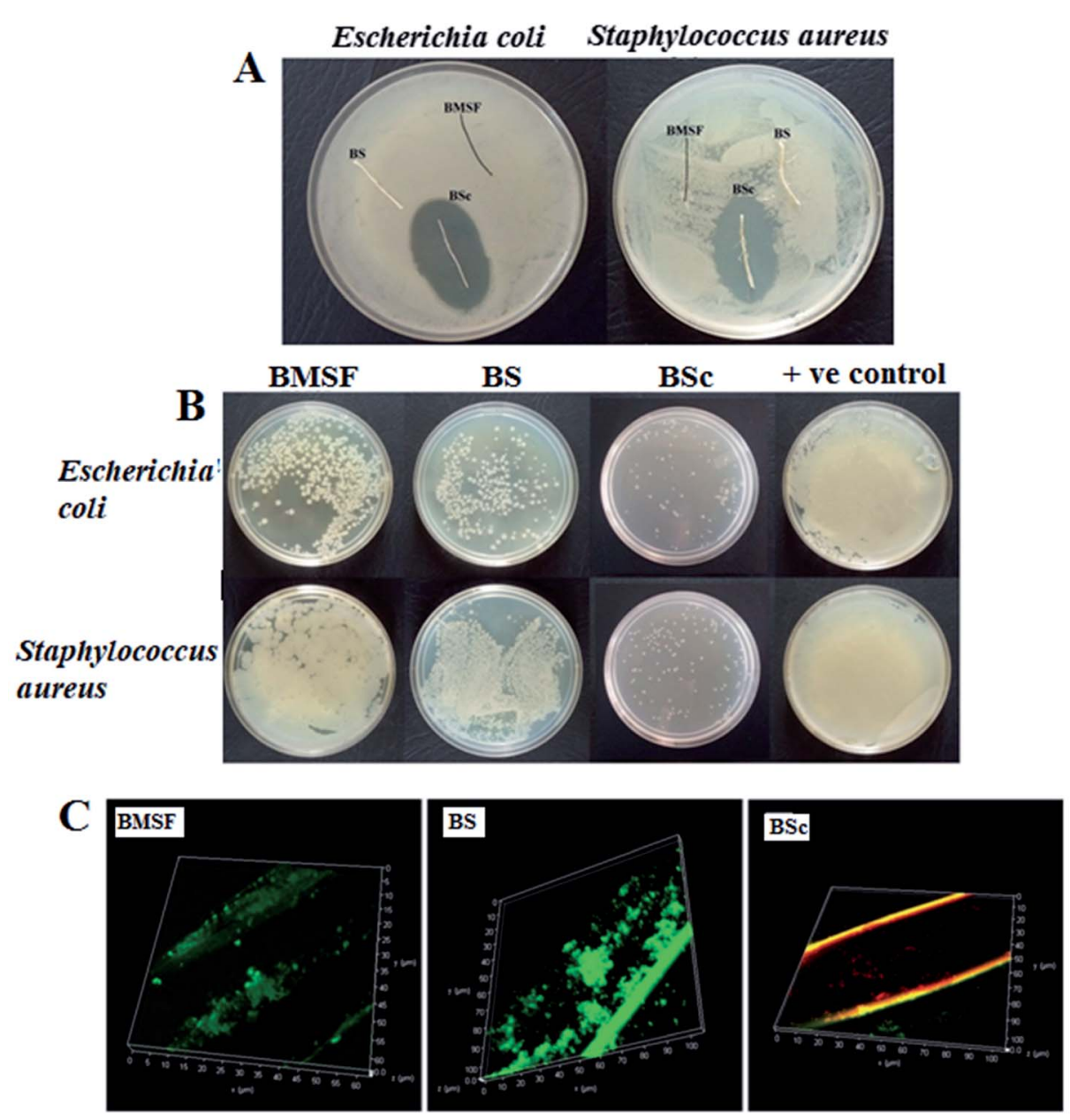

Fig. 3 Photographic images showing antimicrobial activity of BMSF, BS, and BSc against Escherichia coli and Staphylococcus aureus. (A) Standard agar diffusion test method. (B) Re-cultivated colonies of tested bacterial strain on agar plates. (C) Confocal microscopic examination showing viability of the bacteria (S. aureus) on the respective sutures, live bacteria appear green while dead ones are red. 
a broad spectrum antibacterial agent which interrupts the bacterial protein synthesis machinery and is active against various bacterial strains like E. coli, and $S$. aureus. Whereas, clotrimazole acts against a variety of fungal pathogens by disrupting their cell wall permeability. Along with these antimicrobial agents, the natural base materials AV and GA also possess inherent antimicrobial activities. Direct contact of the Gram positive $S$. aureus and Gram negative E. coli with the sutures for 21 days further established the antimicrobial activity of the BMSF, BS, and BSc sutures. Fig. 3B represents the colonies of the re-cultivated bacteria from the BMSF, BS, and BSc treatment groups. The test results clearly showed a lower number of bacterial colonies on the agar plates for BSc, which significantly indicates the poor survival frequency of $E$. coli and $S$. aureus on the plates. The re-cultivated bacterial colonies of BS and BMSF are clearly visualized on the agar medium. The existence of these bacterial colonies implies that bacteria can survive on the BS and BMSF. The presence of the natural (AV and GA) base material and antimicrobial agents (chloramphenicol and clotrimazole) on the BSc prevented the growth of bacteria on the suture, ${ }^{39,40}$

Florescence staining and confocal microscopy analysis was used to visualize and verify the capability of the coating material to fight against viable bacterial colonization and biofilm formation (Fig. 3C). After 21 days of incubation with $S$. aureus, a large number of viable bacteria (green) and an insignificant number of dead bacteria (red) were observed on the BMSF and BS suture surfaces. On the other hand, the number of viable bacteria was significantly lower on the BSc suture surface. The presence of the antimicrobial agents and the natural base material on BSc inhibits the growth of bacteria on the suture surface and prevented biofilm formation.

\subsection{In vitro drug release kinetics}

BSc demonstrated a biphasic release pattern with a slow and sustained release of chloramphenicol for up to $144 \mathrm{~h}$ in all of the tested pH conditions (Fig. 4). It was observed that the drug loaded on BSc was at a concentration of $8 \mu \mathrm{g} \mathrm{cm} \mathrm{cm}^{-1}$ of the suture. At pH 7.7, initially at $24 \mathrm{~h} 35 \pm 3 \%$ drug was released and this gradually increased with the increase in the incubation period. The initial burst release of the loaded drug was due to the loose association of the drug (antimicrobial agent) with the surface of the base material loaded suture. Also, the PBS, which acts as a physiological saline when used as the drug release medium helps the steady release of the drugs to the target site. After incubation for $144 \mathrm{~h}$, the slope of the curve reached a plateau at $87 \pm 3 \%$. Furthermore, at $\mathrm{pH} 6.3$ and 6.8, the suture shows a similar trend in the drug release, in which a release of $70 \pm 3 \%$ and $78 \pm 4 \%$ were observed respectively. The natural base materials, AV and GA used together as the drug loading agent, facilitate the sustained drug release. ${ }^{\mathbf{4 1 , 4 2}} \mathrm{AV}$ assists the drug release mechanism through a biphasic mode; initially by breaking the drug embedded polymeric matrix, followed by diffusion. ${ }^{41}$ Also, gum acacia possesses the ability to hold the loaded drug in the wound area for a longer duration. ${ }^{42}$ To maintain the aseptic condition of the wound for a longer

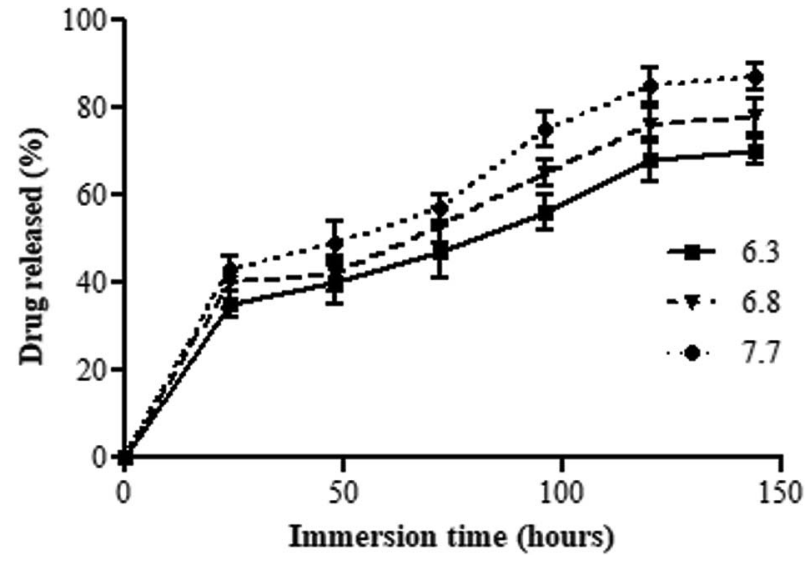

Fig. 4 In vitro drug release percentage of BSc in PBS at three different $\mathrm{pH}$ values of $6.3,6.6$, and 7.7, up to $144 \mathrm{~h}$.

duration of time, sustained release is the most desirable property, which in turn is crucial for accelerated wound healing. ${ }^{22}$ The drug release process starts before the formation of the anatomical barrier and is completed after the wound healing process. $^{\mathbf{4 3 , 4 4}}$ Studies have reported that the alkaline environment accelerates the hydrolytic degradation which enhances the drug release process. ${ }^{45}$ The same result has been achieved in this study by release of the maximum percentage of drug ( $87 \pm$ $3 \%$ ) at alkaline $\mathrm{pH}$ 7.7.

\subsection{Wound healing efficacy analysis}

3.6.1. Surgery. The healing process was observed in all of the three groups of animals sutured with BMSF, BS, and BSc in different post-operative periods (Fig. 5A). In vivo examination revealed distinct differences in the process of wound healing among $S$. aureus infected BMSF, BS, and BSc sutured animals on the seventh post-operative day. Prominent infection was visible in the case of BMSF and the BS sutured group of animals on day seven, whereas no sign of infection and inflammation was observed in the BSc group. On the $14^{\text {th }}$ post-operative day, the wound healing progression was more pronounced in the BSc group as compared to the BS and BMSF groups. The healing process is followed by complete hair growth, without any symptoms of edema and irritation in the healing area. On the other hand, increased edema in the wound area with a purulent discharge was observed for the BS and BMSF stitched animal on the $14^{\text {th }}$ post-surgical day. During the observation period no behavioral differences in the experimental animal groups were observed. Post infection CFU data supported the faster wound healing progression in BSc $(p<0.001)$ sutured animal compared to the BS and BMSF treatment groups (Fig. 5B). The slow and sustained release of chloramphenicol and clotrimazole protects the wound from infections, ${ }^{\mathbf{4 1 , 4 2 , 4 6 , 4 7}}$ which helps with faster wound healing in the BSc sutured animals. The presence of growth factors helped rapid tissue regeneration in the wounded area, whereas the inherent healing properties of the coating material further enhanced the tissue repairing process.

3.6.2. Inflammatory markers. Studies have suggested that traumatic exposure leads to the release of cytokines at the site of 


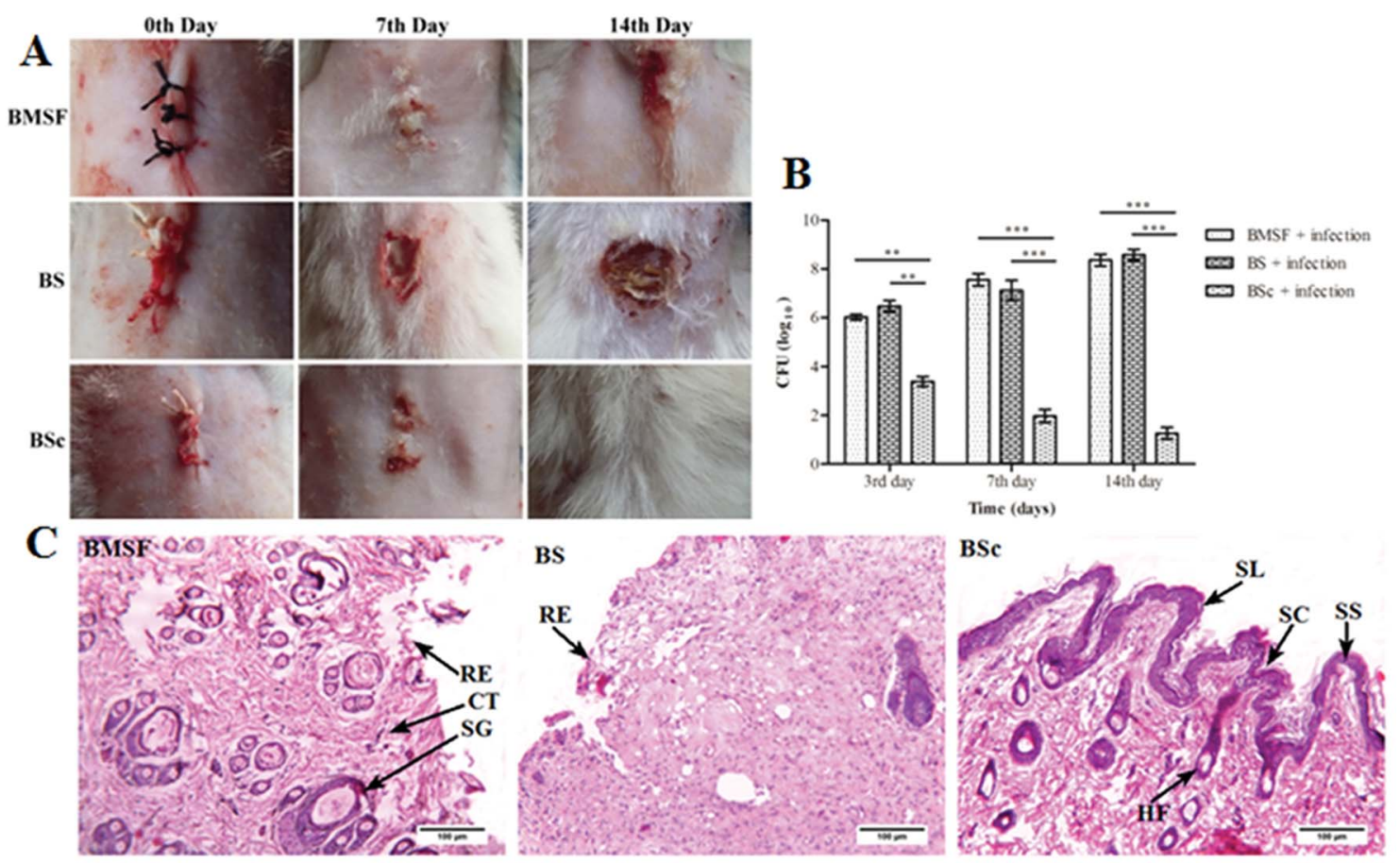

Fig. 5 (A) Healing progression of the S. aureus infected wounds sutured with BMSF, BS, and BSc on day zero, seventh, and $14^{\text {th }}$ day. (B) CFU count data for the incised wound of the BMSF, BS, and BSc sutured animals at different time points. (C) Histopathological observation of the skin tissue sutured with BMSF, BS, and BSc on the $14^{\text {th }}$ post-operative day. Abbreviations: ruptured epithelium (RE); sebaceous gland (SG); collagen tissue $(\mathrm{CT})$; stratum corneum (SC); stratum spisum (SS); and stratum lucidum (SL); and hair follicles (HR).

tissue destruction with subsequent inflammation and trauma. ${ }^{48}$ Infection at the wound site increases the inflammatory markers and prolongs the healing process. ${ }^{49}$ In this study, infected wounds sutured with BS and BMSF showed increased levels of pro-inflammatory cytokines TNF- $\alpha$ and IL-1 $\beta$ on third and seventh post-operative day (Fig. S6, ESI $\dagger$ ). IL-1 $\beta$ activates the neutrophils and fibroblasts, thus stimulating the acuteinflammatory process. TNF- $\alpha$ is a macrophage derived cytokine, produced by the neutrophils. ${ }^{5}$ Thus, TNF- $\alpha$ and IL-1 $\beta$ both play an important role in the initiation of the inflammatory process. On the other hand, the levels of TNF- $\alpha$ and IL-1 $\beta$ were normal $(p<0.001)$ in the infected wounds sutured with BSc on the $14^{\text {th }}$ post-operative day. The presence of the antimicrobial agents, chloramphenicol, clotrimazole, and the natural base material prevented the growth of $S$. aureus in the wound area and promoted the wound healing process.

3.6.3. Histopathology of the wound. To further validate the wound healing efficacy of the BSc suture, histopathological analysis of the skin tissue was performed (Fig. 5C). The results of this study demonstrated that the animals sutured with BSc showed low levels of inflammatory infiltrate and showed pronounced re-epithelialization of the epidermis. Furthermore, the histopathology data revealed that BSc sutured wounds showed complete regeneration of the epidermis with three distinct visible layers, namely the stratum spinosum, stratum corneum, and stratum lucidum, along with normal adnexal structures. Whereas animals sutured with BMSF and BS showed a ruptured epithelium, unorganized collagen tissue, and sebaceous glands. The presence of growth factor helps skin tissue regeneration by the formation of collagen fibers and epidermis in the wounded area. ${ }^{\mathbf{1 7}}$ The nerve growth factor triggers these tissue remodeling functions through the interaction between two classes of cell surface receptors, namely tropomyosin receptor kinase $\mathrm{A}$ and the neurotrophin receptor. ${ }^{2}$ This could have resulted in the faster wound healing in the BSc sutured rats as observed in this study. The antimicrobial properties and tissue growth promoting functionalities of BSc contributed towards the superior wound healing efficacy when compared to the BS and BMSF sutures.

\section{Conclusion}

In the present study, an attempt was made to develop a novel suture biomaterial from the pseudo stem of bananas which is an agricultural byproduct. Furthermore, the suture was functionalized with an AV-GA based hydrogel containing antimicrobial agents and growth factors. The surface modified suture possesses excellent tensile strength along with the desirable physico-chemical properties of an ideal suture. The fabricated suture was found to be biocompatible and also exhibited the sustained release of drugs for up to $144 \mathrm{~h}$. The BSc suture exhibited a significant antimicrobial activity against infectious microbes such as $S$. aureus, E. coli, and C. albicans in both in vitro and in vivo conditions. Furthermore, the BSc sutured animals showed pronounced wound healing through the reduction of infection and related inflammatory markers at the 
wound site. Additionally, the findings of this study could potentially contribute towards the promotion of banana cultivators by adding value to the agricultural waste.

\section{Author contributions}

All of the first authors made a significant contribution towards the conceptualization of the study and agreed with the content of the manuscript. Himadri Kalita and Ankita Hazarika conceived, designed and performed the experiments, analyzed the data and jointly wrote the manuscript. Raghuram Kandimalla and Sanjeeb Kalita performed the biocompatibility studies, antimicrobial assays, in vivo experiments, and contributed towards the data analysis, manuscript writing and corrections. The final approval of the manuscript was done by Rajlakshmi Devi.

\section{Conflicts of interest}

A portion of this work reported in this article was used to file an Indian patent application (201631039603) dated $21^{\text {rd }}$ November 2016.

\section{Acknowledgements}

The authors acknowledge the Department of Science and Technology, Government of India, New Delhi, for financial support and the Institute of Advanced Study in Science and Technology (IASST), Guwahati, Assam, for providing the necessary facilities. We thank Ramie research station, Sarbhog, Assam, India and Mr Haren Medhi for providing the plant fiber. The authors also acknowledge Mr Subrata Goswami, a technical assistant at IASST for evaluating the mechanical properties; Mr Bikash Sarma and Achyut Konwar, PhD scholars at IASST for helping in conducting the FESEM and TGA analysis. All of the authors are grateful to Dr Anupam Banerjee, Manager Application Support, Leica Microsystem and Dr Bula Choudhury, Senior Scientist, Guwahati Biotech Park for their immense help with confocal microscopy.

\section{References}

1 J. Pedro, C. Serrano, L. García-fern, M. Barbeck, S. Ghanaati, R. Unger, J. Kirkpatrick, E. Arzt, L. Funk and P. Tur, Biomaterials, 2015, 52, 291-300.

2 A. Paige, V. Giuseppe and A. Travaglia, J. Inorg. Biochem., 2016, 161, 1-8.

3 C. Justinger, M. R. Moussavian, C. Schlueter, B. Kopp, O. Kollmar and M. K. Schilling, Surgery, 2009, 145, 330-334.

4 K. P. Chellamani, D. Veerasubramanian and R. S. V. Balaji, J. Acad. Ind. Res., 2013, 1, 778-782.

5 R. Kandimalla, S. Kalita, B. Choudhury, D. Devi, D. Kalita, K. Kalita, S. Dash and J. Kotoky, Mater. Sci. Eng., C, 2016, 62, 816-822.

6 N. Venkateshwaran and A. Elayaperumal, J. Reinf. Plast. Compos., 2010, 29, 2387-2396.

7 P. Gañán, J. Cruz, S. Garbizu, A. Arbelaiz and I. Mondragon, J. Appl. Polym. Sci., 2004, 94, 1489-1495.
8 S. Guo and L. A. Dipietro, J. Dent. Res., 2010, 89, 219-229.

9 X. Liu, T. Lin, J. Fang, G. Yao, H. Zhao, M. Dodson and X. Wang, J. Biomed. Mater. Res., Part A, 2010, 94, 499-508.

10 M. Kazemzadeh-Narbat, B. F. L. Lai, C. Ding, J. N. Kizhakkedathu, R. E. W. Hancock and R. Wang, Biomaterials, 2013, 34, 5969-5977.

11 S. Forbes, A. J. McBain, S. Felton-Smith, T. A. Jowitt, H. L. Birchenough and C. B. Dobson, Biomaterials, 2013, 34, 5453-5464.

12 S. Kalita, R. Kandimalla, B. Devi, B. Kalita, K. Kalita, M. Deka, A. Chandra Kataki, A. Sharma and J. Kotoky, RSC Adv., 2017, 7, 1749-1758.

13 F. Kashiwabuchi, K. S. Parikh, R. Omiadze, S. Zhang, L. Luo, H. V Patel, Q. Xu, L. M. Ensign, H.-Q. Mao, J. Hanes and P. J. McDonnell, Transl. Vis. Sci. Technol., 2017, 6, 1.

14 L. Chun-hui, W. Chang-hai, X. Zhi-liang and W. Yi, Process Biochem., 2007, 42, 961-970.

15 A. Konwar, S. Kalita, J. Kotoky and D. Chowdhury, ACS Appl. Mater. Interfaces, 2016, 8, 20625-20634.

16 S. Kalita, R. Kandimalla, A. C. Bhowal, J. Kotoky and S. Kundu, Sci. Rep., 2018, 8, 5778.

17 H. Kalita, A. Hazarika, S. Kalita, R. Kandimalla and R. Devi, RSC Adv., 2017, 7, 32637-32646.

18 A. Jyoti, D. Gogoi, R. Kandimalla, S. Kalita, Y. B. Chaudhari, M. R. Khan, J. Kotoky and J. Chutia, Mater. Sci. Eng. C, 2016, 60, 475-484.

19 J. Li, G. Wang, H. Zhu, M. Zhang, X. Zheng, Z. Di, X. Liu and X. Wang, Sci. Rep., 2014, 4, 4359.

20 A. Konwar, R. Kandimalla, S. Kalita and D. Chowdhury, ACS Sustainable Chem. Eng., 2018, 6, 5806-5817.

21 G. Limbert, R. Bryan, R. Cotton, P. Young, L. Hall-Stoodley, S. Kathju and P. Stoodley, Acta Biomater., 2013, 9, 6641-6652.

22 X. Chen, D. Hou, L. Wang, Q. Zhang, J. Zou and G. Sun, ACS Appl. Mater. Interfaces, 2015, 7, 22394-22403.

23 A. Hazarika, H. Kalita, M. Chandra Kalita and R. Devi, Nutrition, 2017, 38, 95-101.

24 H. Kalita, D. C. Boruah, M. Deori, A. Hazarika, R. Sarma, S. Kumari, R. Kandimalla, J. Kotoky and R. Devi, Front. Pharmacol., 2016, 7, 1-19, DOI: 10.3389/fphar.2016.00102.

25 A. J. Choudhury, D. Gogoi, J. Chutia, R. Kandimalla, S. Kalita, Y. B. Choudhury, M. R. Khan, K. Kalita and J. Kotoky, Surgery, 2016, 159, 539-547.

26 N. Bhardwaj, Y. P. Singh, D. Devi, R. Kandimalla, J. Kotoky and B. B. Mandal, J. Mater. Chem. B, 2016, 4, 3670-3684.

27 A. Hazarika, H. Kalita, D. Chandra, M. Chandra and R. Devi, Nutrition, 2016, 32, 1081-1091.

28 J. T. Kim and A. N. Netravali, Composites, Part A, 2010, 41, 1245-1252.

29 S. Kalia, K. Thakur, A. Celli, M. A. Kiechel and C. L. Schauer, J. Environ. Chem. Eng., 2013, 1, 97-112.

30 V. K. S. M. Moghaddasi Sharrif, Int. J. Biol. Med. Res., 2011, 2, 466-471.

31 F. Al-Rimawi and M. Kharoaf, Chromatogr. Res. Int., 2011, 2011, 1-6.

32 M. Boopalan, M. J. Umapathy and P. Jenyfer, Silicon, 2012, 4, 145-149. 
33 S. Delvasto, E. F. Toro, F. Perdomo and R. M. de Gutiérrez, Constr. Build. Mater., 2010, 24, 187-192.

34 L. S. Kassama, Am. Int. J. Contemp. Res., 2015, 5, 30-39.

35 S. Kalia, B. S. Kaith and I. Kaur, Polym. Eng. Sci., 2009, 49, 1253-1272.

36 V. Mhuka, S. Dube and M. M. Nindi, Int. J. Biol. Macromol., 2013, 52, 305-311.

37 S. Henkelman, G. Rakhorst, J. Blanton and W. van Oeveren, Mater. Sci. Eng., C, 2009, 29, 1650-1654.

38 E. Carolina, D. João, D. Masi, A. Carlos, L. Campos, F. David, J. De Masi, M. Aurelio, S. Ratti, I. Shin, R. David and J. De Mais, Braz. J. Otorhinolaryngol., 2016, 82, 512-521.

39 A. E. Krausz, B. L. Adler, V. Cabral, M. Navati, J. Doerner, R. A. Charafeddine, D. Chandra, H. Liang, L. Gunther, A. Clendaniel, S. Harper, J. M. Friedman, J. D. Nosanchuk and A. J. Friedman, Nanomedicine, 2015, 11, 195-206.

40 K. A. Juby, C. Dwivedi, M. Kumar, S. Kota, H. S. Misra and P. N. Bajaj, Carbohydr. Polym., 2012, 89, 906-913.

41 M. Tummalapalli, M. Berthet, B. Verrier, B. L. Deopura, M. S. Alam and B. Gupta, Int. J. Biol. Macromol., 2016, 82, 104-113.
42 B. A. Aderibigbe, K. Varaprasad, E. R. Sadiku, S. S. Ray, X. Y. Mbianda, M. C. Fotsing, S. J. Owonubi and S. C. Agwuncha, Int. J. Biol. Macromol., 2015, 73, 115-123.

43 T. Dai, M. Tanaka, Y.-Y. Huang and M. R. Hamblin, Expert Rev. Anti-Infect. Ther., 2011, 9, 857-879.

44 G. Gainza, S. Villullas, J. L. Pedraz, R. M. Hernandez and M. Igartua, Nanomedicine, 2015, 11, 1551-1573.

45 M. A. Woodruff and D. W. Hutmacher, Prog. Polym. Sci., 2010, 35, 1217-1256.

46 G. E. Magoulas, O. N. Kostopoulou, T. Garnelis, C. M. Athanassopoulos, G. G. Kournoutou, M. Leotsinidis, G. P. Dinos, D. Papaioannou and D. L. Kalpaxis, Bioorg. Med. Chem., 2015, 23, 3163-3174.

47 S. O. Sequeira, C. A. T. Laia, A. J. L. Phillips, E. J. Cabrita and M. F. Macedo, J. Cult. Herit., 2017, 24, 45-52.

48 G. Schulze-Tanzil, O. Al-Sadi, E. Wiegand, W. Ertel, C. Busch, B. Kohl and T. Pufe, Scand. J. Med. Sci. Sports, 2011, 21, 337351.

49 R. Kandimalla, S. Kalita, B. Choudhury, S. Dash, K. Kalita and J. Kotoky, Front. Pharmacol., 2016, 7, 1-8, DOI: 10.3389/fphar.2016.00198. 